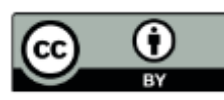

\title{
Toma de decisiones estratégicas en organizaciones de salud. Una revisión de la literatura*
}

\author{
Strategic Decision-Making in Healthcare \\ Organizations. A literature review
}

\section{Tomada de decisões estratégicas em organizações de saúde. Uma revisão da literatura}

Recibido: 20 de Junio de 2019. Aceptado: 13 de Abril de 2020.

Publicado: 1 de Octubre de 2020.

DOI: https://doi.org/10.11144/Javeriana.rgps19.tdeo

\author{
Yuli Licet León Vanegas ${ }^{a}$ \\ Universidad del Rosario, Colombia \\ ORCID: https://orcid.org/0000-0003-4585-9129 \\ Javier Leonardo González-Rodríguez \\ Universidad del Rosario, Colombia \\ ORCID: https://orcid.org/0000-0001-6693-3901
}

\begin{abstract}
Para citar este artículo León YL, González-Rodríguez JL. La toma de decisiones estratégicas en organizaciones de salud. Una revisión de la literatura. Rev Gerenc Polit Salud. 2020;19. https://doi.org/10.11144/ Javeriana.rgps 19.tdeo
\end{abstract}

a Autora de correspondencia. E-mail: yuli.leon@urosario.edu.co 


\section{Resumen}

Introducción. "La toma de decisiones estratégicas es un proceso vital dentro de las organizaciones, debido a las múltiples variables que intervienen, los recursos involucrados y al impacto en el mediano y largo plazo" (1). Objetivo: identificar los factores que influyen en el proceso de toma de decisiones estratégicas en las organizaciones del sector salud. Materiales y método: revisión sistemática de los estudios en el sistema integrado de búsqueda y en las bases de datos electrónicas: Pubmed, EMBASE, ScienceDirect y Google Scholar. La búsqueda se enfocó en artículos empíricos publicados en los últimos 10 años, sin restricción de idioma y con disponibilidad del texto completo. Resultados: se incluyeron 14 artículos que analizaron variables relacionadas con los siguientes factores: tomadores de decisiones, aspectos de la organización y aspectos ambientales externos. Ningún estudio incluyó dentro de su análisis la influencia de los factores específicos de la decisión. Conclusiones: existen diferentes factores contextuales que influyen en el proceso de toma de decisiones estratégicas en organizaciones de salud. No obstante, existen pocos estudios que permitan establecer un marco global que integre las diferentes perspectivas.

Palabras clave: Toma de decisiones, administración en salud, administración de servicios de salud.

\section{Abstract}

Introduction. "Strategic decision-making is a vital process within organizations because of the multiple variables involved, the resources concerned and the impact on the medium and long term" (1). Objective. To identify the factors influencing the strategic decision-making process in health sector organizations. Materials and methods. A systematic review of the studies in the integrated search system and in the electronic databases (Pubmed, EMBASE, ScienceDirect, and Google Scholar) was done. The search was performed for empirical articles published in the last 10 years, without language restriction, and with full-text availability. Results. We included 14 articles analyzing variables related to decision-makers, organizational, and external environmental factors. No study included the influence of the specific factors of the decision within its analysis. Conclusions. There are different contextual factors that influence the strategic decision-making process in health organizations. However, there are few studies that allow us to establish a global framework to integrate the different perspectives.

Keywords: Decision making, health administration, health services administration.

\section{Resumo}

Introdução: “A tomada de decisões estratégicas é um processo vital dentro das organizações, devido às múltiplas variáveis que intervém, os recursos envolvidos e ao impacto no mediano e longo prazo" (1). Objetivo: identificar os fatores que influenciam o processo de tomada de decisões estratégicas nas organizações do setor saúde. Materiais e métodos: revisão sistemática dos estudos no sistema integrado de pesquisa e nas bases de dados eletrônicas: Pubmed, EMBASE, ScienceDirect e Google Scholar. A pesquisa se focou em artigos empíricos publicados nos últimos 10 anos, sem restrição do idioma e com disponibilidade do texto completo. Resultados: incluíram-se 14 artigos que analisaram variáveis relacionadas com os seguintes fatores: tomadores de decisões, aspectos da organização e aspectos ambientais externos. Nenhum estudo incluiu dentro de sua análise a influência dos fatores específicos da decisão. Conclusões: existem diferentes fatores contextuais que influenciam o processo de tomada de decisões estratégicas em organizações de saúde. Contudo, existem poucos estudos que permitam estabelecer um marco global que integre as diferentes perspectivas.

Palavras-chave: Tomada de decisões, administração em saúde e administração, administração de serviços de saúde. 


\section{Introducción}

La toma de decisiones estratégicas tiene sus raíces en el campo de la gestión estratégica en la década de 1960, con los conceptos de estrategia y estructura de Chandler y la estrategia corporativa de Ansoff. Para Chandler, la estrategia es la determinación de las metas y objetivos de una empresa a largo plazo, las acciones a seguir y los recursos necesarios para llevarlos a cabo (2). Por su parte, Andrews conceptualizó la estrategia como el patrón de decisiones en una empresa que determina y plantea sus objetivos, propósitos o metas; además, formula las políticas y planes para la consecución de esos objetivos (3). Cabe destacar que la estrategia permite a una organización hacerle frente a entornos complejos y dinámicos, e implica decisiones que afectan el bienestar a largo plazo de una organización (4).

La toma de decisiones estratégicas constituye un proceso imprescindible para el adecuado funcionamiento de una organización; es considerada como parte importante de la gestión de cualquier organización (5) y, a su vez, la más difícil del trabajo de los directivos (6), por ende, se ha convertido en un aspecto relevante y un motivo de preocupación central para las organizaciones modernas (7), debido a sus implicaciones para la adaptación y la asimilación del cambio, su alta influencia en el éxito o el fracaso empresarial (8) y la creación de valor (9). Adicionalmente, pueden requerir gran cantidad de recursos (10) y poseen un efecto multiplicador, dado que afectan no solo a la organización en la que se toman, sino también a la sociedad (11).

Por otro lado, Mintzberg, Raisinghani y Theoret establecieron que una decisión estratégica es "importante, en términos de las acciones tomadas, los recursos comprometidos o los precedentes establecidos" (12). Asimismo, las decisiones son tomadas por los líderes de una organización y afectan de manera crítica la salud y supervivencia de la misma (13); para tomarlas se requiere de un proceso por medio del cual se analizan las diferentes alternativas, a fin de reducir la incertidumbre sobre el logro de un resultado deseado, con el mejor efecto sobre la organización (14).

En las últimas décadas, la investigación en el proceso de toma de decisiones ha crecido teórica y empíricamente $(15,16)$, lo que evidencia la influencia de diferentes factores contextuales $(17,18,19)$, y hace referencia a una multiplicidad de variables que intervienen en el proceso de toma de decisiones, las cuales se han clasificado en cuatro categorías principales: las específicas de la decisión, las características de la organización, las ambientales externas y las características individuales de los directivos (17). En consecuencia, estos factores se abordan en la literatura a través de cuatro perspectivas diferentes: la elección estratégica, el determinismo ambiental, las características de la empresa y las características específicas de la decisión (16).

La perspectiva de la elección estratégica. Esta se centra en el papel y los atributos de los tomadores de determinaciones y hace hincapié en que las decisiones estratégicas obedecen, en parte, al comportamiento endógeno y, por tanto, reflejan la idiosincrasia de los primeros $(20,21)$. 
De acuerdo con Schwenk, las características individuales afectan los mapas heurísticos y cognitivos que se utilizan para tomar decisiones (22). En este sentido, propuso tres categorías de variables de diferencias individuales: el estilo cognitivo, los factores demográficos y los rasgos de personalidad. Bajo esta perspectiva, Child postuló que los principales tomadores de disposiciones tienen un control considerable sobre la dirección futura de la organización (20).

Desde la teoría de los niveles superiores, Hambrick y Mason sugirieron que las características demográficas de los administradores conllevan una base cognitiva y de valores al proceso de toma de decisiones que restringe su campo de visión, de manera que son "potentes predictores de las estrategias" (23). Del mismo modo, argumentaron que mientras quienes adoptan las determinaciones estén expuestos a un flujo continuo de estímulos posibles, estas bases cognitivas y de valores distorsionan la percepción de quien adopta esas decisiones y, por ello, afectan la decisión estratégica.

La perspectiva del determinismo ambiental. De acuerdo con el determinismo ambiental, los procesos de toma de decisiones estratégicas expresan la adaptación a las oportunidades, las amenazas, las restricciones y otras características del medio ambiente (24), lo cual implica que las condiciones ambientales dominantes dejan un escaso margen de maniobra a los responsables. Por tal motivo, los gerentes no pueden alterar sustancialmente el curso de la organización. Estudios empíricos han determinado que variables del entorno, como el dinamismo y la hostilidad, ejercen un efecto estadísticamente significativo sobre las dimensiones del proceso de toma de decisiones estratégicas $(15,25)$.

La perspectiva de las características de la empresa. La literatura de gestión estratégica menciona que las características de la organización influyen en el proceso de toma de decisiones (26). En este sentido, se ha sugerido que los procesos de toma de decisiones estratégicas se ven afectados por una variedad de factores organizacionales como la estructura, la distribución de poder, las estrategias pasadas, los sistemas internos, el tamaño, el control corporativo y el desempeño (27). Papadakis, Lioukas y Chambers encontraron que las características internas de las empresas tienen efectos más significativos sobre el proceso de toma de decisiones estratégicas que las variables ambientales (28).

Las características específicas de la decisión. Además de las perspectivas anteriores, la literatura establece que las características propias de la decisión tienen un impacto considerable en los procesos de toma de decisiones (29). Diferentes autores sugirieron que el mismo estímulo interno o externo puede ser interpretado de varias formas por los gerentes en distintas organizaciones o, incluso, dentro de la misma organización $(17,30)$. Estos argumentaron que la manera en que los administradores categorizan y etiquetan una decisión en las primeras etapas del proceso influye fuertemente en las respuestas subsiguientes. 
Elbanna, Ali y Dayan llegaron a la conclusión de que más que los factores externos e internos, son las características específicas de la decisión las que determinan el proceso (31). Por su parte, Simon sostuvo que es probable que la naturaleza del problema a resolver sea el determinante principal del grado en que los responsables de las decisiones utilizan procesos racionales y/o intuitivos en la toma de estas (32).

Las empresas se desempeñan en un entorno que plantea desafíos y retos de diversa índole, por tanto, el proceso de toma de decisiones ejerce un papel fundamental para la supervivencia de la organización y es allí donde los factores contextuales que lo influyen son relevantes para cualquier tipo de organización, siendo vitales en ambientes dinámicos y organizaciones complejas como las instituciones de salud, pues la falta de adaptación a los cambios se ha asociado con el fracaso en la industria hospitalaria (33).

Es de señalar que el sector salud se desenvuelve en un entorno altamente dinámico, caracterizado por una creciente competencia y una necesaria contención de costos, debido a los cambios de las tendencias sociales en las últimas décadas, la demografía poblacional, las innovaciones tecnológicas, el aumento de la regulación estatal y los intereses en conflicto de los diferentes actores involucrados. Adicionalmente, deben ofrecer mejores resultados y una atención oportuna y de alta calidad (34). Lo anterior ha ocasionado que el cambio al que se enfrentan las organizaciones de cuidado de la salud sea más acelerado, en comparación con el que ocurre en muchos otros sectores (35).

Por tales razones, las instituciones de salud deben perseguir múltiples objetivos estratégicos de calidad en la práctica clínica, investigación, enseñanza, rentabilidad y eficiencia de los recursos y concordancia con la legislación vigente. Esto conlleva a que los administradores de las organizaciones del sector se enfrenten a una gran presión en la toma de decisiones para el cumplimiento de estos objetivos, sin dejar de lado ninguno (36).

Esta complejidad del sector del cuidado de la salud requiere un mayor énfasis en la toma de decisiones estratégicas para mejorar el desempeño (37), de ahí que las determinaciones adoptadas por los administradores y directores ejecutivos definen la competitividad relativa en la prestación de servicios a la sociedad (20). En el presente artículo se realizó una revisión de la literatura con el propósito de identificar los factores que influyeron en el proceso de toma de decisiones estratégicas en las organizaciones del sector salud en los últimos diez años.

\section{Metodología}

\section{Estrategia de búsqueda}

Se llevó a cabo una revisión sistemática de los estudios que analizan la toma de decisiones estratégicas en organizaciones de salud. Para esto se hizo una investigación en el sistema integrado de búsqueda de la Universidad del Rosario, que permite el acceso a todas las bases de datos, contenidos (electrónicos e impresos), a través de un único cuadro de búsqueda y 
en las siguientes bases de datos electrónicas relevantes: Pubmed, EMBASE y ScienceDirect, complementado con una búsqueda libre en Google Scholar.

La búsqueda consistió en una combinación de términos relacionados para las siguientes características: el constructo de interés (Strategic decision making) y objeto de estudio (healthcare), ver la figura 1. En dicha búsqueda se priorizaron artículos empíricos publicados en la última década, sin restricción de idioma y con disponibilidad de texto completo. Se excluyeron editoriales, estudios narrativos, comentarios y conferencias.

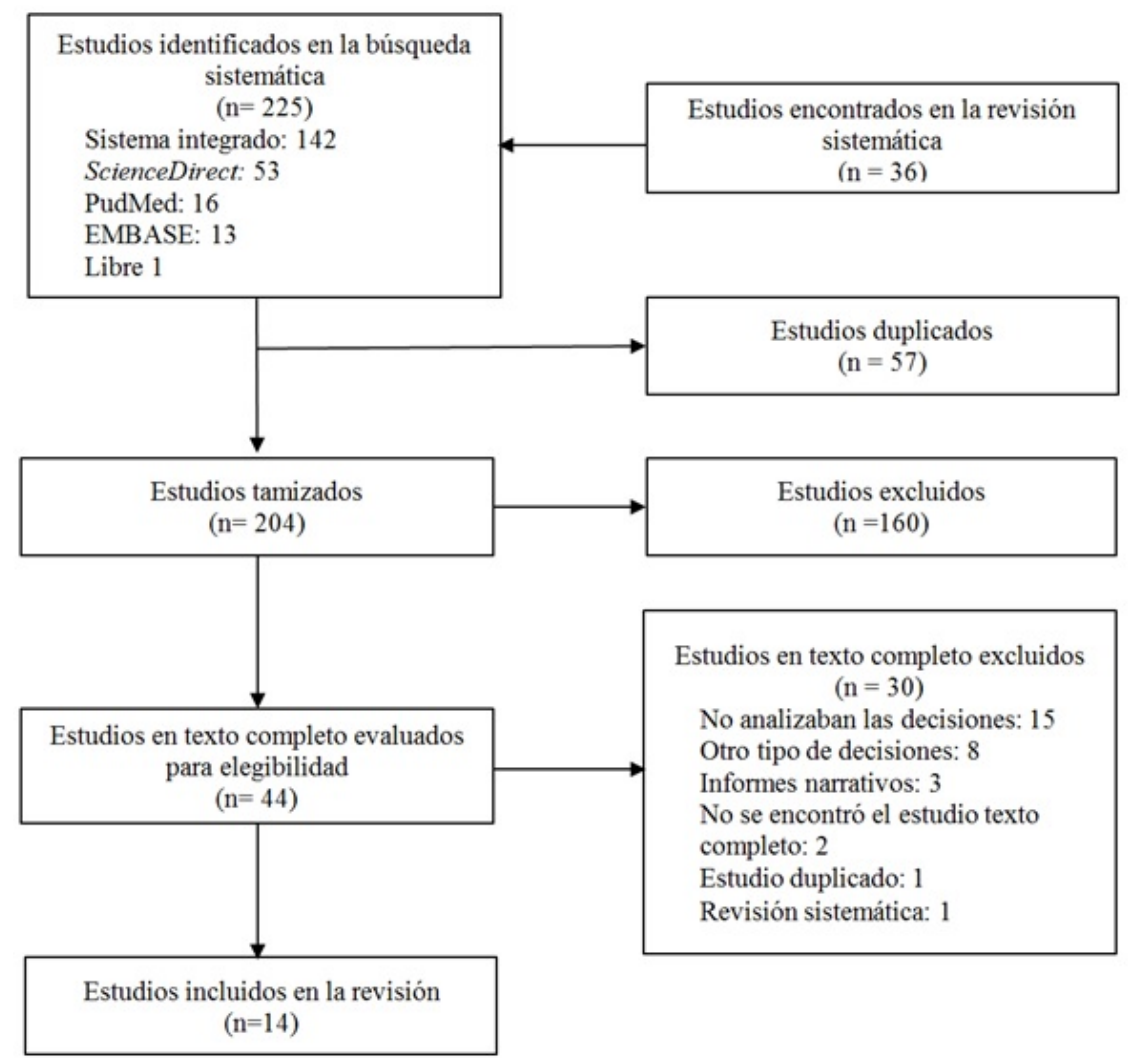

Figura 1 Diagrama de flujo para la selección de estudios Fuente: Prisma, elaboración propia. 


\section{Selección de estudios}

Para la selección de estudios se establecieron los siguientes criterios de elegibilidad: se definió como tema de investigación las decisiones estratégicas en organizaciones de salud. Se excluyeron los trabajos que abordan el proceso desde una perspectiva clínica, operativa o a nivel de salud pública. De manera que solo se incluyeron en el análisis los artículos que hablan de la toma de decisiones desde un punto de vista gerencial. La selección de estudios se determinó a partir de la revisión del título, palabras clave y resumen; los que cumplían con los criterios de elegibilidad continuaron para el proceso de revisión en texto completo.

\section{Extracción de datos}

Los artículos se analizaron en texto completo para identificar cómo las investigaciones abordaron los componentes establecidos. Las variables se definieron para extraer la información de interés, para lo cual se desarrolló una plantilla que incluye los datos del estudio, el objetivo, el tipo de decisión estratégica, los participantes o la muestra, los tipos de métodos utilizados para la recopilación y el análisis de datos, los factores influyentes, el país y las principales conclusiones.

Para capturar la medida en que los componentes se han estudiado e identificar enfoques comunes se extrajeron y sintetizaron las variables utilizadas para el análisis de datos. No se definió un conjunto fijo de categorías para cada componente.

La identificación de los estudios, la extracción de datos y la síntesis fueron llevadas a cabo por el primer autor. El procedimiento fue auditado por un investigador principal, para garantizar la idoneidad de los componentes, la estrategia de búsqueda, el proceso de selección de estudios y para aclarar las decisiones sobre las publicaciones, cuyos criterios de inclusión/exclusión eran ambiguos.

\section{Resultados}

Se incluyeron 261 estudios, de los cuales se estableció una referencia fuera de la búsqueda sistemática; 36 trabajos fueron identificados de la revisión realizada por Akyürek et al. (14). La Figura 1 proporciona un diagrama de flujo de la selección del análisis (38). Después de una tamización por título y resumen, se revisaron 44 artículos en texto completo, de los cuales 14 cumplieron con los criterios de elegibilidad. Los artículos que han sido revisados en texto completo, pero excluidos fueron categorizados por las razones que llevaron a tomar esta última determinación. La mayoría de los estudios no analizaba las decisiones en sí mismas (15): examinaban otro tipo de resoluciones (8) o correspondían a informes narrativos (3).

Finalmente, se incluyeron 14 artículos, cuyas características se presentan en la tabla 1. La mayoría de los trabajos se originaron en Estados Unidos (9) y Finlandia (2), seguidos por Reino Unido, Canadá e Irán con uno, respectivamente; todos se publicaron en inglés. Dentro de las principales estrategias para la recopilación de datos utilizadas se encuentran la encuesta (8) y la entrevista (6); los principales participantes fueron altos directivos y ejecutivos. 
Yuli Licet León Vanegas / Javier Leonardo González-Rodríguez

\section{Tabla 1 Estudios seleccionados}

\begin{tabular}{|c|c|c|c|c|}
\hline Estudio & País & Objetivo & Participantes & $\begin{array}{c}\text { Diseño / } \\
\text { Recopilación de } \\
\text { datos }\end{array}$ \\
\hline $\begin{array}{l}\text { Kash, Spaulding, } \\
\text { Gamm y Johnson } \\
(2014) .(39)\end{array}$ & EE. UU. & $\begin{array}{l}\text { Mejorar la comprensión del proceso de } \\
\text { decisión estratégica, las iniciativas } \\
\text { estratégicas y el comportamiento de } \\
\text { gestión relacionado con la } \\
\text { implementación de la estrategia en los } \\
\text { hospitales. }\end{array}$ & $\begin{array}{l}61 \text { administradores } \\
\text { de atención médica. }\end{array}$ & $\begin{array}{l}\text { Cualitativo, estudio } \\
\text { de caso comparativo. } \\
\text { Entrevistas } \\
\text { semiestructuradas. }\end{array}$ \\
\hline $\begin{array}{l}\text { Langabeer y } \\
\text { DelliFraine } \\
\text { (2011). (40) }\end{array}$ & EE. UU. & $\begin{array}{l}\text { Examinar si el optimismo sirve como } \\
\text { un sesgo cognitivo que cortocircuita el } \\
\text { proceso estratégico o, específicamente, } \\
\text { si da como resultado un mayor uso del } \\
\text { incrementalismo frente a un proceso } \\
\text { racional integral. }\end{array}$ & $\begin{array}{l}\text { Directores } \\
\text { ejecutivos de } 168 \\
\text { hospitales. }\end{array}$ & $\begin{array}{l}\text { Cuantitativo } \\
\text { transversal, } \\
\text { descriptivo, } \\
\text { correlacional. } \\
\text { Encuestas. }\end{array}$ \\
\hline $\begin{array}{l}\text { Langabeer y Yao } \\
(2012) .(41)\end{array}$ & EE. UU. & $\begin{array}{l}\text { Explorar el impacto que tiene el } \\
\text { optimismo disposicional en la relación } \\
\text { bien establecida entre los procesos } \\
\text { racionales de toma de decisiones y el } \\
\text { desempeño organizacional. }\end{array}$ & $\begin{array}{l}168 \text { hospitales con } \\
\text { fines de lucro de } \\
\text { EE. UU. }\end{array}$ & $\begin{array}{l}\text { Cuantitativo } \\
\text { descriptivo } \\
\text { correlacional. } \\
\text { Encuestas }\end{array}$ \\
\hline $\begin{array}{l}\text { Parayitam (2010). } \\
(42)\end{array}$ & EE. UU. & $\begin{array}{l}\text { Investigar empíricamente si la } \\
\text { confianza basada en la competencia } \\
\text { entre los ejecutivos médicos y los } \\
\text { administradores es beneficiosa para las } \\
\text { organizaciones de atención médica. }\end{array}$ & $\begin{array}{l}\text { Equipos de alta } \\
\text { gerencia de } 109 \\
\text { hospitales. }\end{array}$ & $\begin{array}{l}\text { Cuantitativo } \\
\text { descriptivo, } \\
\text { correlacional. } \\
\text { Encuestas. }\end{array}$ \\
\hline $\begin{array}{l}\text { Parayitam, Phelps } \\
\text { y Olson (2007). } \\
\text { (43) }\end{array}$ & EE. UU. & $\begin{array}{l}\text { Examinar empíricamente los resultados } \\
\text { de las decisiones, cuando los ejecutivos } \\
\text { médicos participaron en el proceso de } \\
\text { toma de determinaciones estratégicas } \\
\text { en las organizaciones de atención } \\
\text { médica. }\end{array}$ & $\begin{array}{l}361 \text { ejecutivos de } \\
109 \text { hospitales }\end{array}$ & $\begin{array}{l}\text { Cuantitativo } \\
\text { descriptivo, } \\
\text { correlacional. } \\
\text { Encuestas. }\end{array}$ \\
\hline $\begin{array}{l}\text { Parayitam y } \\
\text { Dooley (2007). } \\
\text { (44) }\end{array}$ & EE. UU. & $\begin{array}{l}\text { Demostrar los roles divergentes de la } \\
\text { confiabilidad percibida como } \\
\text { moderadores potenciales en los equipos } \\
\text { estratégicos de toma de decisiones. }\end{array}$ & $\begin{array}{l}\text { CEO y altos } \\
\text { ejecutivos de } 109 \\
\text { hospitales }\end{array}$ & $\begin{array}{l}\text { Cuantitativo } \\
\text { descriptivo, } \\
\text { correlacional. } \\
\text { Encuestas. }\end{array}$ \\
\hline $\begin{array}{l}\text { Parayitam y } \\
\text { Dooley (2011). } \\
(45)\end{array}$ & EE. UU. & $\begin{array}{l}\text { Examinar la relación entre el conflicto } \\
\text { cognitivo en los resultados de la } \\
\text { decisión mientras controla el conflicto } \\
\text { afectivo. }\end{array}$ & $\begin{array}{l}109 \text { hospitales. } 254 \\
\text { encuestados } \\
\text { individualmente. }\end{array}$ & $\begin{array}{l}\text { Cuantitativo } \\
\text { descriptivo, } \\
\text { correlacional. } \\
\text { Encuestas. }\end{array}$ \\
\hline $\begin{array}{l}\text { Shoemaker, } \\
\text { Kazley y White } \\
(2010) .(46)\end{array}$ & EE. UU. & $\begin{array}{l}\text { Describir el proceso de toma de } \\
\text { decisiones organizativas utilizado en la } \\
\text { determinación de los conceptos de } \\
\text { diseño basado en la evidencia, los } \\
\text { criterios utilizados y la medida en que } \\
\text { el estilo de liderazgo puede haber } \\
\text { influido en el proceso de toma de } \\
\text { decisión. }\end{array}$ & $\begin{array}{l}\text { Comunidades } \\
\text { Californianas y sus } \\
\text { Centros de Salud }\end{array}$ & $\begin{array}{l}\text { Mixto, estudio de } \\
\text { caso. } \\
\text { Entrevistas, } \\
\text { observación } \\
\text { participante, revisión } \\
\text { de pruebas } \\
\text { documentales y } \\
\text { cuestionario. }\end{array}$ \\
\hline
\end{tabular}

Fuente: elaboración propia. 
Toma de decisiones estratégicas en organizaciones de salud. Una revisión de la literatura

Tabla 1 Estudios seleccionados (Cont.)

\begin{tabular}{|c|c|c|c|c|}
\hline Estudio & País & Objetivo & Participantes & $\begin{array}{c}\text { Diseño / } \\
\text { Recopilación de } \\
\text { datos }\end{array}$ \\
\hline $\begin{array}{l}\text { Sosnowy, Weiss, } \\
\text { Maylahn, Pirani y } \\
\text { Katagiri (2013). } \\
\text { (47) }\end{array}$ & EE. UU. & $\begin{array}{l}\text { Establecer el uso de los procesos de } \\
\text { toma de decisiones por parte de los } \\
\text { líderes de los departamentos de salud } \\
\text { locales del estado de Nueva York y el } \\
\text { personal de nivel superior e identificar } \\
\text { facilitadores y barreras para el uso de la } \\
\text { toma de determinaciones basados en la } \\
\text { evidencia. }\end{array}$ & $\begin{array}{l}31 \text { departamentos } \\
\text { de salud locales. } 47 \\
\text { participantes }\end{array}$ & $\begin{array}{l}\text { Cualitativo. } \\
\text { Entrevistas y grupos } \\
\text { focales. }\end{array}$ \\
\hline $\begin{array}{l}\text { Kallio y } \\
\text { Kuoppakangas } \\
(2013) .(48)\end{array}$ & Finlandia & $\begin{array}{l}\text { Comprender el fenómeno relacionado } \\
\text { con el reciente efecto de arrastre de las } \\
\text { empresas municipales en Finlandia y, } \\
\text { especialmente, el razonamiento } \\
\text { relacionado con la adopción de la } \\
\text { forma de empresa municipal. }\end{array}$ & $\begin{array}{l}3 \text { instituciones } \\
\text { hospitalarias. }\end{array}$ & $\begin{array}{l}\text { Cualitativo, estudio } \\
\text { de caso múltiple. } \\
\text { Entrevistas. }\end{array}$ \\
\hline $\begin{array}{l}\text { Simonen, } \\
\text { Viitanen, Lehto y } \\
\text { Koivisto, A. M. } \\
(2009) .(49)\end{array}$ & Finlandia & $\begin{array}{l}\text { Investigar cómo los gerentes de } \\
\text { servicios sociales y de salud evalúan } \\
\text { las fuentes de conocimiento que } \\
\text { afectan su toma de decisiones y cómo } \\
\text { las evaluaciones se asociaron con los } \\
\text { antecedentes profesionales, el sector de } \\
\text { actividad, el género, la edad y la } \\
\text { experiencia gerencial de los directivos. }\end{array}$ & $\begin{array}{l}404 \text { encuestados de } \\
\text { un hospital } \\
\text { universitario. }\end{array}$ & $\begin{array}{l}\text { Cuantitativo. } \\
\text { Encuesta. }\end{array}$ \\
\hline $\begin{array}{l}\text { Siddiqui, Ramesh, } \\
\text { Manoharan, } \\
\text { Hussein, Jawad y } \\
\text { Hussain (2014). } \\
\text { (50) }\end{array}$ & $\begin{array}{l}\text { Reino } \\
\text { Unido }\end{array}$ & $\begin{array}{l}\text { Identificar las consideraciones } \\
\text { estratégicas clave que contribuyen a la } \\
\text { expansión exitosa y si estas pudieran } \\
\text { ser utilizadas como parte de un marco } \\
\text { para ayudar a informar el proceso de } \\
\text { toma de decisiones de los institutos } \\
\text { británicos que contemplan, en el futuro, } \\
\text { la internacionalización. }\end{array}$ & $\begin{array}{l}\text { Dos instituciones de } \\
\text { salud. }\end{array}$ & $\begin{array}{l}\text { Análisis cualitativo } \\
\text { exploratorio. } \\
\text { Entrevista } \\
\text { semiestructuradas y } \\
\text { análisis de la } \\
\text { documentación. }\end{array}$ \\
\hline $\begin{array}{l}\text { MacDonald, Bath } \\
\text { y Booth (2008). } \\
(51)\end{array}$ & Canadá & $\begin{array}{l}\text { Examinar la información que los } \\
\text { gerentes de servicios de salud } \\
\text { utilizaron para respaldar decisiones que } \\
\text { no están relacionadas con la atención } \\
\text { individual del paciente. }\end{array}$ & $\begin{array}{l}19 \text { altos ejecutivos, } \\
\text { directores y } \\
\text { gerentes. }\end{array}$ & $\begin{array}{l}\text { Cualitativo, estudio } \\
\text { de caso múltiple, } \\
\text { exploratorio. } \\
\text { Entrevista } \\
\text { semiestructurada. }\end{array}$ \\
\hline $\begin{array}{l}\text { Lashgari, } \\
\text { Antuchevičiené, } \\
\text { Delavari, y } \\
\text { Kheirkhah (2014). } \\
\text { (52) }\end{array}$ & Irán & $\begin{array}{l}\text { Evaluar en diferentes aspectos los } \\
\text { factores internos y externos que afectan } \\
\text { la tercerización de los servicios de } \\
\text { salud en el diputado de salud del } \\
\text { TUMS. }\end{array}$ & Gerentes y expertos & $\begin{array}{l}\text { Cuantitativo } \\
\text { descriptivo. } \\
\text { Encuestas. }\end{array}$ \\
\hline
\end{tabular}

Fuente: elaboración propia.

Cabe señalar que ocho estudios mencionaron el tipo de decisiones estratégicas identificadas, las cuales se muestran consolidadas y resumidas en la tabla 2. Dentro de ellas están el desarrollo de nuevos planes o enfoques estratégicos, la construcción de nuevas sedes o expansiones, la compra o el traslado del hospital o sucursal, la apertura de nuevos servicios o programas, las reestructuraciones y la implementación de planes de mejora de la calidad y seguridad, y de servicio al cliente. 
Tabla 2 Tipos de decisiones

\begin{tabular}{|l|c|c|}
\hline \multicolumn{1}{|c|}{ Tipos de decisiones } & $\mathrm{N}^{\circ}$ & Estudio \\
\hline $\begin{array}{l}\text { Relacionadas con el enfoque estratégico de la } \\
\text { organización (por ej. alianzas, } \\
\text { internacionalización, tercerización, nuevos } \\
\text { mercados, cambio de naturaleza). }\end{array}$ & 7 & $(39,42,43,44,48,50,52)$ \\
\hline $\begin{array}{l}\text { Construcción de nuevas sedes/ampliación de } \\
\text { servicios o instalaciones. }\end{array}$ & 5 & $(39,42,43,44,47)$ \\
\hline Compra o traslado de un hospital/sucursal. & 4 & $(39,42,43,44)$ \\
\hline Apertura de nuevos servicios o programas. & 4 & $(39,42,43,44)$ \\
\hline Reestructuración de departamentos o servicios. & 4 & $(39,42,43,44)$ \\
\hline Planes de mejora del servicio al cliente. & 4 & $(39,42,43,44)$ \\
\hline $\begin{array}{l}\text { Programas de mejora en la calidad y seguridad } \\
\text { del paciente. }\end{array}$ & 4 & $(39,42,43,44)$ \\
\hline Cierre de programas o servicios. & 3 & $(42,43,44)$ \\
\hline Reclutamiento de médicos y enfermeras. & 3 & $(42,43,44)$ \\
\hline $\begin{array}{l}\text { Iniciativas para mejorar la gestión de los } \\
\text { recursos/rentabilidad. }\end{array}$ & 2 & $(39,42)$ \\
\hline Adquisición de nuevos equipos/sistemas. & 2 & $(39,42)$ \\
\hline $\begin{array}{l}\text { Mejoras en la contratación/beneficios al } \\
\text { personal. }\end{array}$ & 2 & $(39,42)$ \\
\hline Reducción de la fuerza laboral. & 1 & $(42)$ \\
\hline Venta de un hospital. & $(42)$ \\
\hline
\end{tabular}

Fuente: elaboración propia

Posteriormente, para cada estudio se clasificaron las variables que influyeron en la toma de decisiones estratégicas. Para ello, se escogió el marco establecido por Dean y Sharfman (17), quienes las agruparon en cuatro tipos de factores contextuales: factores del tomador de decisiones, factores de la organización, factores ambientales externos y factores específicos de la decisión.

Por otro lado, nueve estudios analizaron variables relacionadas con uno de los factores, cuatro estudios analizaron dos y solo un estudio incluyó variables de tres factores. Por su parte, los elementos relacionados con las características del tomador de decisiones y los relacionados con la organización fueron los más estudiados, seguidos por los factores ambientales externos. Ningún estudio contempló dentro de su análisis de la adopción de decisiones la influencia de los factores específicos de la decisión (ver tabla 3 ). 
Posteriormente, para cada estudio se clasificaron las variables que influyeron en la toma de decisiones estratégicas. Para ello, se escogió el marco establecido por Dean y Sharfman (17), quienes las agruparon en cuatro tipos de factores contextuales: factores del tomador de decisiones, factores de la organización, factores ambientales externos y factores específicos de la decisión.

Por otro lado, nueve estudios analizaron variables relacionadas con uno de los factores, cuatro estudios analizaron dos y solo un estudio incluyó variables de tres factores. Por su parte, los elementos relacionados con las características del tomador de decisiones y los relacionados con la organización fueron los más estudiados, seguidos por los factores ambientales externos. Ningún estudio contempló dentro de su análisis de la adopción de decisiones la influencia de los factores específicos de la decisión (ver tabla 3).

Tabla 3 Factores contextuales

\begin{tabular}{|l|c|c|}
\hline \multicolumn{1}{|c|}{ Factores } & $\mathrm{N}^{\circ}$ & Estudio \\
\hline Factores del tomador de decisiones & 7 & $(40-45,49)$ \\
\hline Factores de la organización & 7 & $(46-52)$ \\
\hline Factores ambientales externos & 6 & $(39,46,49-52)$ \\
\hline Factores especificos de la decisión & 0 & -- \\
\hline
\end{tabular}

Fuente: elaboración propia. 
Yuli Licet León Vanegas / Javier Leonardo González-Rodríguez

\section{Discusión}

La revisión de la literatura realizada sobre los factores que influyen en la toma de decisiones estratégicas en organizaciones de salud muestra cómo la investigación empírica ha abordado el tema, si bien no se pudo establecer un enfoque general de análisis, se identificaron varias conclusiones e implicaciones para futuros estudios.

En primer lugar, la investigación en el proceso de toma de decisiones ha aumentado teórica y empíricamente en los últimos años, lo que evidencia la influencia de diferentes factores contextuales en el proceso de toma de desiciones estrategicas (17-19). Sin embargo, como lo muestra esta revisión, la investigación en las organizaciones de salud aún es incipiente, teniendo en cuenta que el entorno de atención de la salud es diferente al de otras industrias, debido, principalmente, a la dificultad de definir y medir el producto, el alto grado de coordinación entre los grupos profesionales, la ambigüedad y/o conflicto de los roles derivados de las dos líneas de autoridad (médica y administrativa), y los complicados vínculos entre sistemas e intersistemas (53).

A esto se suman las grandes transformaciones que ha sufrido la industria en los últimos tiempos, derivadas de los cambios en la tecnología, la epidemiología de la población y la política económica y regulatoria, y de los múltiples actores involucrados en el proceso de atención. Todo esto ha convertido a la industria de la salud en un entorno amenazante, de mayor competencia y contención de costos.

En segundo lugar, respecto al alcance de los estudios, la investigación se centró en analizar, en su mayoría, variables de un solo un grupo de factores que influyen en la toma de decisiones. Diferentes autores establecieron que al incluir variables de cada uno de los cuatro factores contextuales se pueden comparar el impacto global y el poder predictivo de cada uno, para explicar la variación en los procesos de adopción de decisiones estratégicas y sus implicaciones (16,28,54-56), en especial, incluir las características específicas de la decisión, que en esta revisión no se encontró ningún estudio que las incluyera y las cuales se han establecido que tienen un impacto considerable en los procesos de toma de determinaciones, pues el mismo estímulo interno o externo puede ser interpretado de varias maneras por los gerentes en distintas organizaciones o incluso dentro de la misma organización $(17,30)$.

En tercer lugar, la mayoría de los estudios fueron realizados en Estados Unidos. Existe una cantidad limitada de investigación llevada a cabo en otros países y ninguna identificada en el contexto latinoamericano, lo que suma al debate la aplicabilidad de los resultados obtenidos a otras regiones $(28,56,57)$. También, este último hecho plantea la necesidad de investigar el proceso de toma de decisiones estratégicas en los países de América Latina, para no solo comprender mejor este fenómeno sino comparar resultados e identificar posibles convergencias y diferencias. 
Por último, es necesario resaltar que los estudios, directa o indirectamente, enmarcan la importancia de la información en la toma de decisiones estratégicas como un insumo esencial para seleccionar la alternativa más razonable en la consecución de los objetivos establecidos. Esto es consecuente con la investigación en el área que establece la racionalidad entendida como la "medida en que el proceso de decisión involucra la recolección de información relevante para la decisión y su análisis para hacer la elección” (17), como una de las principales dimensiones del proceso de toma de decisiones estratégicas.

\section{Conclusión}

La literatura revisada para la elaboración de este documento permitió evidenciar que existen diferentes factores contextuales que influyen en el proceso de toma de decisiones estratégicas en organizaciones de salud. Empero, la escasez de estudios existentes no permite diseñar un marco global que integre las diferentes perspectivas. De acuerdo con ello, las investigaciones futuras deberían centrarse en incluir variables correspondientes a cada uno de los cuatro factores contextuales, para comparar el impacto global y contribuir a una mejor comprensión de lo que influye en el proceso de toma de decisiones estratégicas.

\section{Agradecimientos}

Financiado en el marco del Doctorado en Ciencias de la Dirección de la Universidad del Rosario. Agradecimientos a la Universidad del Rosario.

\section{Referencias}

1. XIV Coloquio Internacional. La influencia del contexto en el proceso de toma de decisiones estratégicas en organizaciones de salud. Lima: Cladea Editorial; s.f.

2. Chandler A. Strategy and structure: Chapters in the history of the industrial enterprise, $2^{\text {nd }}$ ed. Massachusetts: Cambridge, Mass: MIT Press; 1990.

3. Andrews K. The concept of corporate strategy, 1st ed. Richard D. Irwin (ed.) New York: McGraw-Hill School Education Group; 1980.

4. Hambrick D. Some tests of the effectiveness and functional attributes of miles and snow's strategic types. Acad Manag J. 1983;26(1): 5-26. https://doi.org/10.2307/256132.

5. Plunkett WR, Attner RF. Introduction to management, $3^{\text {rd }}$ ed. London: International Thomson Publishing; 1992.

6. Mark S. Delaying decisions stifles: industrial management decision-making progress. Management Decision. 1997;45: 1622-1635. https://doi.org/10.1016/j.jbusres.2011.08.006

7. Mitchell JR, Shepherd DA, Sharfman MP. Erratic strategic decisions: when and why managers are inconsistent in strategic decision making. Strateg Manag J. 2011;32(7): 683-704. https://doi.org/10 $.1002 / \mathrm{smj} .905$ 
8. Thompson AA, Strickland AJ. Strategic management: Concepts and cases. Chicago: Irwin Publishing Co.; 1995.

9. Rodríguez E, Pedraja L. Análisis del impacto del proceso de toma de decisiones estratégicas sobre la eficacia de las organizaciones públicas. Innovar. 2009;19(35): 33-46. https://revistas.unal.edu.co/in dex.php/innovar/article/view/28707

10. Hofer CW, Schendel D. Strategy formulation: Analytical concepts, $1^{\text {st }}$ ed. South-Western; 1978.

11. Colignon R, Cray D. Critical organizations. Organ Stud. 1980;1(4): 349-365. https://doi.org/10.1177/ 017084068000100403

12. Mintzberg H, Raisinghani D, Theoret A. The structure of "unstructured" decision processes. Admin Sci Q. 1976;21(2): 246-275. https://doi.org/10.2307/2392045

13. Eisenhardt KM, Zbaracki MJ. Strategic decision making. Strateg Manag J. 1992;13(2): 17-37. https:/ /doi.org/10.1002/smj.4250130904

14. Akyürek ÇE, Sawalha R, Ide S. Factors affecting the decision making process in healthcare institutions. Acad Strateg Manag J. 2015;14(1): 1-14. https://www.abacademies.org/articles/asmj-volume-14-sp ecial-issue.pdf\#page $=5$

15. Elbanna S, Thanos IC, Papadakis VM. Understanding how the contextual variables influence political behaviour in strategic decision-making: a constructive replication. J Strateg Manag. 2014;7(3): 226-250. https://doi.org/10.1108/JSMA-02-2014-0013

16. Rajagopalan N, Rasheed AM, Datta DK. Strategic decision processes: Critical review and future directions. J Manag. 1993;19(2): 349-384. https://doi.org/10.1016/0149-2063(93)90057-T

17. Dean JW, Sharfman MP. Procedural rationality in the strategic decision-Making process. J Manag Stud. 1993;30(4): 587-610. https://doi.org/10.1111/j.1467-6486.1993.tb00317.x

18. Priem RL, Rasheed AMA, Kotulic AG. Rationality in strategic decision processes, environmental dynamism and firm performance. J Manag. 1995;21(5): 913-929. https://doi.org/10.1016/0149-206 3(95)90047-0.

19. Elbanna S, Child J. The influence of decision, environmental and firm characteristics on the rationality of strategic decision-making. J Manag Stud. 2007;44(4): 561-591. https://doi.org/10.1111/j.1467-6 486.2006.00670.x

20. Child J. Organizational structure, environment and performance: The role of strategic choice. Sociology. 1972;6(1): 1-22. https://doi.org/10.1177/003803857200600101

21. Cyert R, March JG. A behavioural theory of the firm. Primera ed. New Jersey: Englewood Cliffs; 1963.

22. Schwenk CR. The cognitive perspective on strategic decision making. J Manag Stud. 1988;25(1): 41-55. https://doi.org/10.1111/j.1467-6486.1988.tb00021.x

23. Hambrick DC, Mason PA. Upper Echelons: The organization as a reflection of its top managers. Acad Manag Rev. 1984;9(2): 193-206. https://doi.org/10.2307/258434 
24. Hannan MT, Freeman J. The population ecology of organizations. Am J Sociol. 1977;82(5): 929-964. https://www.jstor.org/stable/2777807

25. Meissner P, Wulf T. Antecendents and effects of decision comprehensiveness: the role of decision quality and perceived uncertainty. Europ Manag J. 2014;32(4): 625-635. https://doi.org/10.1016/j.emj.201 3.10 .006

26. Sutcliffe KM, McNamara G. Controlling decision-Making practice in organizations. Organ Sci. 2001;12(4): 484-501. https://www.jstor.org/stable/3085984.

27. Romanelli E, Tushman ML. Inertia, environments and strategic choice: A quasi-experimental design for comparative-longitudinal research. Manag Sci. 1986;32(5): 608-621. https://doi.org/10.1287/mn sc.32.5.608.

28. Papadakis V, Lioukas S, Chambers D. Strategic decision-making processes: The role of management and context. Strateg Manag J. 1998;19(2): 115-147. https://doi.org/10.1002/(SICI)1097-0266(19980 2)19:2115::AID-SMJ9413.0.CO;2-5.

29. Hickson DJ. Top decisions: strategic decision-making in organizations, $1^{\text {st }}$ ed. San Francisco: JosseyBas; 1986.

30. Dutton JE, Fahey L, Narayanan VK. Toward understanding strategic issue diagnosis. Strateg Manag J. 1983;4(4): 307-323. https://doi.org/10.1002/smj.4250040403

31. Elbanna S, Ali AJ, Dayan M. Conflict in strategic decision making: do the setting and environment matter? International J Confl Manag. 2011;22(3): 278-299. https://doi.org/10.1108/1044406111115 2973

32. Simon HA. Rationality as process and as product of thought. Am Econ Rev. 1978;68(2): 1-16. https:/ /www.jstor.org/stable/1816653

33. Arnould RJ, DeBrock LM. Competition and market failure in the hospital industry: A review of the evidence. Med Care Res Rev. 1986;43(2): 253-292. https://doi.org/10.1177/107755878604300203

34. Ginter P, Duncan WJ, Swayne LE. The strategic management of health care organizations, $1^{\text {st }}$ ed. New York: John Wiley \& Sons; 2009.

35. McConnell CR. The changing face of health care management. Health Care Manag. 2000;18(3): 1-17. https://doi.org/10.1097/00126450-200018030-00002.

36. Hanson L, Carey T, Caprio A, Lee T, Ersek M, Garrett J, et al. Improving decision-making for feeding options in advanced dementia: a randomized, controlled trial. J Am Geriatr Soc. 2011;59(11): 2009-2016. https://doi.org/10.1111/j.1532-5415.2011.03629.x.

37. Alexander JA, Hearld LR, Jiang HJ, Fraser I. Increasing the relevance of research to health care managers: Hospital CEO imperatives for improving quality and lowering costs. Health Care Manag Rev. 2007;32(2): 150-159. https://doi.org/10.1097/01.HMR.0000267792.09686.e3

38. Cochrane Iberoamérica. Manual Cochrane de revisiones sistemáticas de intervenciones.; 2012. http:// www.cochrane.es/?q=es/node/269. 
39. Kash B, Spaulding A, Gamm LD, Johnson CE. Healthcare strategic management and the resource based view. Journal of Strategy and Management. 2014;7(3): 251-264. https://doi.org/10.1108/JSMA-062013-0040

40. Langabeer JR, DelliFraine J. Does CEO optimism affect strategic process? Manag Res Rev. 2011;34(8): 857-868. https://doi.org/10.1108/01409171111152484.

41. Langabeer JR, Yao E. The impact of chief executive officer optimism on hospital strategic decision making. Health Care Manag Rev. 2012;37(4): 310-319. https://doi.org/10.1097/HMR.0b013e31823 $5243 b$

42. Parayitam S. The effect of competence - based trust between physicians and administrative executives in healthcare on decision outcomes. Manag Res Rev. 2010;33(2): 174-191.https://doi.org/10.1108/0 1409171011015856

43. Parayitam S, Phelps LD, Olson BJ. Strategic decision-making in the healthcare industry: the effects of physician executives on decision outcomes. Manag Res News. 2007;30(4): 283-301. https://doi.org /10.1108/01409170710736329

44. Parayitam S, Dooley RS. The relationship between conflict and decision outcomes: moderating effects of cognitive- and affect-based trust in strategic decision-making teams. Intern J Confl Manag. 2007;18(1):42-73. https://doi.org/10.1108/10444060710759318.

45. Parayitam S, Dooley RS. Is too much cognitive conflict in strategic decision-making teams too bad? Intern J Confl Manag. 2011;22(4): 342-357. https://doi.org/10.1108/10444061111171350.

46. Shoemaker L, Kazley A, White A. Making the case for evidence-based design in healthcare: a descriptive case study of organizational decision making. HERD. 2010;4(1): 56-88. https://doi.org/10.1177/19 3758671000400105

47. Sosnowy CD, Weiss LJ, Maylahn CM, Pirani SJ, Katagiri NJ. Factors affecting evidence-based decision making in local health departments. Am J Prev Med. 2013;45(6): 763-768. https://doi.org/10.1016 /j.amepre.2013.08.004

48. Kallio T, Kuoppakangas P. Bandwagoning municipal enterprises: Institutional isomorphism and the search for the Third Way. Policy Studies. 2013;34(1): 19-35. https://doi.org/10.1080/01442872.201 2.731842

49. Simonen O, Viitanen E, Lehto J, Koivisto A. Knowledge sources affecting decision-making among social and health care managers. J Health Organ Manag. 2009;23(2): 183-199. https://doi.org/10.11 $08 / 14777260910960920$

50. Siddiqui S, Ramesh A, Manoharan K, Hussein A, Jawad AM, Hussain F. Developing a framework for the internationalization of british healthcare institutes: A qualitative dual case study analysis. Intern J Healthcare Manag. 2014;7(1): 14-20. https://doi.org/10.1179/2047971913Y.0000000059.

51. MacDonald J, Bath P, Booth A. Healthcare services managers: what Information do they need and use? Evid Based Libr Inf Pract. 2008;3(3): 18-38. https://doi.org/10.18438/B8Q90C. 
52. Lashgari S, Antuchevičienė J, Delavari A, Kheirkhah O. Using QSPM and WASPAS methods for determining outsourcing strategies. JOEBM. 2014;15(4): 1-15. https://doi.org/10.3846/16111699.2 014.908789

53. Walters BA, Clarke L, HES, Shandiz M. Strategic decision-Making among top executives in acute-care hospitals. Health Mark Q. 2001;19(1): 43-59. https://doi.org/10.1300/J026v19n01_04

54. Child J, Chung L, Davies H. The performance of cross-Border units in China: a test of natural selection, strategic choice and contingency theories. J Int Bus Stud. 2003;34(3): 242-254. https://doi.org/10.1 057/palgrave.jibs. 8400033

55. Shepherd NG, Rudd JM. The influence of context on the strategic decision-Making process: A review of the literature. Int J Manag Rev. 2014;16(3): 340-364. https://doi.org/10.1111/ijmr.12023

56. Elbanna S, Child J. Influences on strategic decision effectiveness: development and test of an integrative model. Strateg Manag J. 2007;28(4): 431-453. https://doi.org/10.1002/smj.597

57. Schwenk CR. Strategic Decision Making. J Manag. 1995;21(3): 471-493. https://doi.org/10.1177/014 920639502100304 


\section{Anexos}

Tabla A1 Protocolo de búsqueda

\begin{tabular}{|c|c|}
\hline Pregunta & $\begin{array}{l}\text { ¿Cuáles son los factores que influyen en la toma de } \\
\text { decisiones estratégicas en las organizaciones de salud? }\end{array}$ \\
\hline Bases de datos & $\begin{array}{l}\text { Búsqueda en el sistema integrado de búsqueda de la } \\
\text { Universidad del Rosario, que permite el acceso a todas las } \\
\text { bases de datos, contenidos (electrónicos e impresos) a través } \\
\text { de un único cuadro de búsqueda y en estas bases de datos } \\
\text { electrónicas: Pubmed, EMBASE y ScienceDirect, } \\
\text { complementado con una búsqueda libre en Google Scholar y } \\
\text { búsqueda manual en bola de nieve. }\end{array}$ \\
\hline Tipo de publicación & $\begin{array}{l}\text { Artículos científicos publicados y disponibles en texto } \\
\text { completo. }\end{array}$ \\
\hline Lenguaje & Sin restricción de idioma. \\
\hline Periodo de búsqueda & $2008-2018$ \\
\hline Criterios de inclusión & $\begin{array}{l}\text { Artículos empíricos o teóricos que abordan el tema de la } \\
\text { toma de decisiones estratégicas en organizaciones de salud, } \\
\text { en el cual analicen la influencia de los factores contextuales. }\end{array}$ \\
\hline Términos de búsqueda & $\begin{array}{l}\text { Las estrategias de búsqueda emplearon como términos clave } \\
\text { Strategic decision-making, healthcare Ecuación Strategic } \\
\text { decision-making, healthcare. }\end{array}$ \\
\hline $\begin{array}{l}\text { Tamización de } \\
\text { referencias y } \\
\text { selección de } \\
\text { estudios }\end{array}$ & $\begin{array}{l}\text { La tamización de referencias se basa en título y resumen, } \\
\text { para identificar los artículos potencialmente relevantes, } \\
\text { seguido de la selección de los estudios verificando los } \\
\text { criterios de elegibilidad en el texto completo de las } \\
\text { referencias preseleccionadas. Los resultados se resumen } \\
\text { mediante el diagrama PRISMA. }\end{array}$ \\
\hline $\begin{array}{l}\text { Extracción y } \\
\text { sintesis de datos }\end{array}$ & $\begin{array}{l}\text { Se desarrolló una plantilla que incluye los datos del estudio, } \\
\text { objetivo, tipo de decisión estratégica, participantes o } \\
\text { muestra, los tipos de métodos utilizados para la recopilación } \\
\text { y el análisis de datos, los factores influyentes, el país y las } \\
\text { principales conclusiones. }\end{array}$ \\
\hline
\end{tabular}

Fuente: elaboración propia.

Notas

Artículo de revisión. 